\title{
$\beta$-alanine supplementation improves in-vivo fresh and fatigued skeletal muscle relaxation speed
}

\author{
Rebecca Louise Jones $^{1} \cdot$ Cleveland Thomas Barnett $^{1} \cdot$ Joel Davidson $^{1} \cdot$ Billy Maritza $^{1}$. \\ William D. Fraser ${ }^{2,3} \cdot$ Roger Harris $^{4} \cdot$ Craig Sale $^{1}$
}

Received: 17 October 2016 / Accepted: 11 February 2017 / Published online: 27 March 2017

(c) The Author(s) 2017. This article is an open access publication

\begin{abstract}
Purpose In fresh muscle, supplementation with the ratelimiting precursor of carnosine, $\beta$-alanine (BA), results in a decline in muscle half-relaxation time (HRT) potentially via alterations to calcium $\left(\mathrm{Ca}^{2+}\right)$ handling. Accumulation of hydrogen cation $\left(\mathrm{H}^{+}\right)$has been shown to impact $\mathrm{Ca}^{2+}$ signalling during muscular contraction, carnosine has the potential to serve as a cytoplasmic regulator of $\mathrm{Ca}^{2+}$ and $\mathrm{H}^{+}$coupling, since it binds to both ions. The present study examined the effect of BA supplementation on intrinsic in-vivo isometric knee extensor force production and muscle contractility in both fresh and fatigued human skeletal muscle assessed during voluntary and electrically evoked (nerve and superficial muscle stimulation) contractions.

Methods Twenty-three males completed two experimental sessions, pre- and post- 28 day supplementation with 6.4 g.day $^{-1}$ of BA $(n=12)$ or placebo (PLA; $\left.n=11\right)$. Isometric force was recorded during a series of voluntary and electrically evoked knee extensor contractions.
\end{abstract}

Communicated by Nicolas Place.

Craig Sale

Craig.Sale@ntu.ac.uk

1 Musculoskeletal Physiology Research Group, Sport, Health and Performance Enhancement (SHAPE) Research Centre, School of Science and Technology, Nottingham Trent University, Erasmus Darwin Building, Clifton Lane, Clifton, Nottingham NG11 8NS, UK

2 Norwich Medical School, University of East Anglia, Norwich, Norfolk, UK

3 Norfolk and Norwich University Hospital, Norwich, Norfolk, UK

4 Junipa Ltd, Newmarket, Suffolk, UK
Results BA supplementation had no effect on voluntary or electrically evoked isometric force production, or twitch electromechanical delay and time-to-peak tension. There was a significant decline in muscle HRT in fresh and fatigued muscle conditions during both resting $(3 \pm 13 \%$; $19 \pm 26 \%)$ and potentiated $(1 \pm 15 \% ; 2 \pm 20 \%)$ twitch contractions.

Conclusions The mechanism for reduced HRT in fresh and fatigued skeletal muscle following BA supplementation is unclear. Due to the importance of muscle relaxation on total energy consumption, especially during short, repeated contractions, BA supplementation may prove to be beneficial in minimising contractile slowing induced by fatigue.

Trial registration The trial is registered with Clinicaltrials.gov, ID number NCT02819505.

Keywords Contractile properties - Electrical stimulation . Muscle fatigue $\cdot$ Carnosine

Abbreviations

$\eta_{g}^{2} \quad$ Generalised eta squared

$\eta_{p}^{2} \quad$ Partial eta squared

ANOVA Analysis of variance

BA $\quad \beta$-alanine

$\mathrm{Ca}^{2+} \quad$ Calcium

$\mathrm{CV} \quad$ Coefficient of variation

EMD Electromechanical delay

EMG Surface electromyography

$\mathrm{H}^{+} \quad$ Hydrogen cation

HRT Half-relaxation time

$\mathrm{M}_{\max } \quad$ The mean M-wave area of the three supramaximal stimuli

M-wave Muscle action potential

MVIC Maximal voluntary isometric contraction

MVIF Maximal voluntary isometric force 


$\begin{array}{ll}\text { PLA } & \text { Placebo } \\ \text { RF } & \text { Rectus femoris } \\ \text { RMS } & \text { Root mean squared } \\ \text { TPT } & \text { Time-to-peak tension } \\ \text { TTF } & \text { Time-to-task failure } \\ \text { VL } & \text { Vastus lateralis } \\ \text { VM } & \text { Vastus medialis }\end{array}$

\section{Introduction}

Carnosine ( $\beta$-alanyl- ${ }_{L}$-histidine) is a cytoplasmic dipeptide, synthesised from $\beta$-alanine (BA) and histidine and is found in high concentrations in skeletal muscle. The synthesis of carnosine is limited by the availability of BA from the diet, while supplementation with BA over a number of weeks results in significant increases in the skeletal muscle carnosine content (Harris et al. 2006; Hill et al. 2007). BA supplementation has been shown to consistently increase human skeletal muscle carnosine concentrations, and by an equal amount in both type I and II muscle fibres (Hill et al. 2007), with increases of 40-80\% evident depending upon dose (3.2-6.4 $\mathrm{g} \mathrm{day}^{-1}$ ) and duration of administration (4-10 weeks) (Harris et al. 2006; Hill et al. 2007). Increasing skeletal muscle carnosine concentrations via BA supplementation in both upper and lower limbs has consistently been shown to benefit high-intensity exercise capacity and performance, as highlighted by several reviews (Sale et al. 2010, 2013) and a recent meta-analysis (Hobson et al. 2012).

With a pKa of 6.83 for the histidine imidazole ring when combined with BA and the abundance of carnosine within skeletal muscle, it has been proposed that the improvements in high-intensity exercise outcomes following BA supplementation are the result of increased muscle buffering capacity over the exercise $\mathrm{pH}$ transit range (Hill et al. 2007). Whilst the role of carnosine as an intracellular $\mathrm{pH}$ buffer is undisputable, other physiological roles for carnosine underlying high-intensity exercise improvements following BA supplementation have been proposed (Sale et al. 2010). Carnosine can increase the sensitivity of the calcium $\left(\mathrm{Ca}^{2+}\right)$ release channels in the sarcoplasmic reticulum and/ or the sensitivity of the contractile apparatus in chemically skinned muscle fibres from frogs (Lamont and Miller 1992), mechanically skinned rat muscle fibres (Dutka and Lamb 2004) and type I and type II human skeletal muscle fibres (Dutka et al. 2012). Muscle $\mathrm{Ca}^{2+}$ release channels contain saturable binding sites for carnosine, indicating that carnosine has the potential to alter the $\mathrm{Ca}^{2+}$ channel itself (Batrukova and Rubstov 1997). Until recently, however, research has been limited to rodent and in-vitro models.

Recent work (Hannah et al. 2015) examined the effect of BA supplementation on human skeletal muscle contractile properties and force production capabilities in-vivo. BA supplementation did not alter maximal or explosive voluntary isometric force production, or the force-frequency relationship (Hannah et al. 2015), this relationship is the in-vivo analogue of the force-calcium concentration relationship (Batrukova and Rubstov 1997). These findings were in-line with the hypothesis arising from our previous exercise performance studies (Sale et al. 2010, 2013) proposing that the main physiological role for carnosine in improving high-intensity exercise performance related to intracellular $\mathrm{pH}$ buffering and not to increased $\mathrm{Ca}^{2+}$ sensitivity of the in-vivo contractile apparatus. An unexpected result was a significant decline in half-relaxation time (HRT) during both resting and potentiated twitches following BA supplementation, making it important to confirm these findings before exploring potential underlying mechanisms. Muscle relaxation speed can be impacted by the rate of: (1) dissociation of $\mathrm{Ca}^{2+}$ from troponin (Little et al. 2011); (2) the rate of translocation of $\mathrm{Ca}^{2+}$ to near the site of entry into the sarcoplasmic reticulum (Muntener et al. 1995); (3) reuptake of $\mathrm{Ca}^{2+}$ into the sarcoplasmic reticulum by $\mathrm{Ca}^{2+}$ pumps (Nogueira et al. 2013) and cross-bridge detachment (Allen et al. 2008). Slowing of skeletal muscle relaxation decreases power output and shortening velocity (Allen et al. 2008), thus limiting performance during dynamic exercise where rapidly alternating movements are performed (Allen et al. 1995). Improving the relaxation of skeletal muscle can be energetically beneficial by increasing the efficiency of joint movements by reduced co-contraction (Nogueira et al. 2013). This would be expected to contribute to enhanced dynamic exercise performance, especially where rapidly alternating movements are performed.

High-intensity exercise leads to a more pronounced accumulation of hydrogen cation $\left(\mathrm{H}^{+}\right)$, a metabolic factor which might be involved in skeletal muscle fatigue, but in combination with other fatigue-induced changes or in an indirect manner (Westerblad 2016). It should also be highlighted that muscle fatigue is multi-factorial phenomenon. Skeletal muscle fatigue is generally accompanied by a marked slowing of relaxation (Allen et al. 2008). $\mathrm{H}^{+}$are proposed to directly or indirectly inhibit sarcoplasmic $\mathrm{Ca}^{2+}$ release during skeletal muscle contraction (Laver et al. 2000, 2004). Carnosine has the potential to serve as a cytoplasmic regulator of $\mathrm{Ca}^{2+}$ and $\mathrm{H}^{+}$coupling, since it binds to both ions (Baran 2000). As such, it could be hypothesised that increasing muscle carnosine content, via BA supplementation, would have a more pronounced beneficial effect on HRT when the muscle is fatigued (Bergstrom and Hultman 1988).

The present study aimed to examine the effects of 28 days of BA supplementation on intrinsic in-vivo isometric 
knee extensor force production and muscle contractility in both fresh (rested conditions) and fatigued human skeletal muscle.

\section{Methods}

\section{Ethical approval}

All participants were fully informed of any risks and discomforts associated with the study. Participants provided written informed consent and completed a health screen questionnaire prior to taking part in the study, which was first approved by the Nottingham Trent University Ethical Advisory Committee.

\section{Participants}

Twenty-four male participants were allocated to the two supplement groups [placebo (PLA) or BA] on the basis of maximal voluntary isometric force (MVIF) values recorded during familiarisation. One participant withdrew from the study (PLA group; $n=11$ ) with no reason provided, and consequently, 23 participants completed all aspects of the study (PLA; age, $22 \pm 1$ years, height, $1.83 \pm 0.06 \mathrm{~m}$, body mass, $81.4 \pm 14.2 \mathrm{~kg}$, MVIF, $600 \pm 149 \mathrm{~N}$; and $\mathrm{BA}$; age, $22 \pm 2$ years, height, $1.80 \pm 0.05 \mathrm{~m}$, body mass, $76.0 \pm 7.3 \mathrm{~kg}$, MVIF, $565 \pm 86 \mathrm{~N}$ ). Participants had not ingested any nutritional supplements, had no injuries of the lower limb, and were not involved in any systematic physical training in the 6 months prior to the study. Participants were requested to maintain similar levels of physical activity and dietary intake, which was verbally confirmed at the start of each session. None of the participants were vegetarian or vegan, and therefore, they would likely have encountered small amounts of BA in their diet.

\section{Study design}

This was a double-blind, placebo-controlled study with all raw data analyses, exclusions, and statistical analyses undertaken blind to the supplement group. Participants undertook three experimental sessions; a familiarisation session, which preceded a baseline session by $\sim 7$ days, and a follow-up session after 28 days of supplementation. Participants were instructed to abstain from alcohol and strenuous/unaccustomed exercise for $36 \mathrm{~h}$ before measurement sessions, with caffeine prohibited on the day of testing. Compliance with these requests was confirmed verbally with participants before commencing each session. Measurement sessions recorded force and surface electromyography (EMG) during a series of voluntary and involuntary (electrically evoked) isometric contractions of the knee extensors of the dominant leg. All participants were first familiarised with the protocol measures, both baseline, and follow-up sessions involved an identical protocol performed according to a strict schedule.

\section{Supplementation}

Participants were provided with $6.4 \mathrm{~g} \mathrm{day}^{-1}$ of either BA (sustained-release CarnoSyn ${ }^{\mathrm{TM}}$; NAI, Inc. San Marcos, USA) or a matched PLA (maltodextrin; NAI, Inc. San Marcos, USA) for 28 days $(2 \times 800 \mathrm{mg}$ tablets, ingested 4 times per day). Based on similar BA supplementation protocols muscle carnosine content was expected to increase to $\sim 38 \mathrm{mmol} \cdot \mathrm{kg}^{-1}$ dry muscle (based upon a $65 \%$ increase from a baseline concentration of $23 \mathrm{mmol} \cdot \mathrm{kg}^{-1}$ dry muscle) or $65 \%$ above the typical carnosine content of individual eating a mixed diet (Harris et al. 2006; Sale et al. 2013). The sustained-release formulation used in this study has been shown to reduce or remove the paraesthesia often experienced by participants following doses of free BA powder (Decombaz et al. 2012). Supplement compliance was verified with participant logs. Compliance was similar in both groups and was reported as $91 \pm 6 \%$ (BA) and $92 \pm 9 \%$ (PLA; independent sample $t$ test, $P=0.68$ ); no feelings of paraesthesia were reported. Supplements were provided in identical white tubs by an individual blind to the supplement groups. BA tablets were tested by the manufacturer before release for the study and conformed to the label claim for BA content. To ensure no contamination with steroids or stimulants according to the International Organization for Standardization (IOS) 17,025 accredited tests, the BA and PLA supplements were independently tested by HFL Sports Science.

\section{Experimental setup}

The experimental setup for the determination of isometric knee extension force, EMG, and electrical stimulation in our laboratory has been described in detail previously (Hannah et al. 2015).

\section{Isometric knee extension force}

The participant was strapped into a custom-built dynamometer with knee and hip joint angles of $\sim 95$ and $100^{\circ}\left(180^{\circ}\right.$ $=$ full extension), and with an ankle cuff attached $\sim 2 \mathrm{~cm}$ proximal to the medial malleolus secured around the participant's dominant leg, and which was in series with a linear strain gauge (Model 615; Tedea-Huntleigh, Herzliya, Israel). The chair position, strain gauge position, and strapping setup were recorded during the familiarisation session and replicated identically during subsequent testing sessions. Force signals were amplified $(\times 1,000)$ in the 
frequency range of $0-500 \mathrm{~Hz}$, sampled at $2000 \mathrm{~Hz}$ using an external A/D converter (Model 1401; CED, Cambridge, UK), interfaced with a personal computer (PC) using the Spike 2 software (CED). Force data were low-pass filtered in both directions at $450 \mathrm{~Hz}$ using a fourth-order zero-lag Butterworth filter before analysis. Baseline resting force was subtracted from all force recordings to correct for the effects of gravity.

\section{Electromyography}

EMG signals were recorded from the superficial quadriceps: $m$. rectus femoris (RF), $m$. vastus medialis (VM), and $m$. vastus lateralis (VL). EMG signals were pre-amplified by active EMG leads (input impedance: $100 \mathrm{M} \Omega$; common mode rejection ratio: $>100 \mathrm{~dB}$; base gain: 500; first order high-pass filter set to $10 \mathrm{~Hz}$; Noraxon, Scottsdale, AR) connected in series to a custom-built junction box and subsequently to the same analogue-digital converter and PC software that enabled synchronisation with the force data. The signals were sampled at $2000 \mathrm{~Hz}$. EMG data were band-pass filtered in both directions between 20 and $450 \mathrm{~Hz}$ using a fourth-order zero-lag Butterworth filter before analysis.

\section{Electrical stimulation}

Knee extensor contractile properties were assessed using a constant current variable voltage stimulator (DS7AH; Digitimer, Welwyn Garden City, UK). Square-wave pulses (0.2 ms duration) were delivered via: (1) supramaximal femoral nerve stimulation to evoke maximal resting twitch, potentiated twitch, and octet contractions; and (2) percutaneous submaximal muscle stimulation to evoke contractions at a range of frequencies ( 1 to $100 \mathrm{~Hz}$ ) to assess the force-frequency relationship. A cathode stimulation probe (1 cm diameter; Electro-Medical Supplies, Wantage, UK) and an anode $(7 \times 10 \mathrm{~cm}$ carbon rubber electrode; Electro- Medical Supplies) were used to elicit femoral nerve stimulation. Two carbon rubber electrodes $(14 \times 10 \mathrm{~cm}$; Electro-Medical Supplies) were used to elicit percutaneous stimulation.

\section{Protocol and measurements}

Identification of force and EMG onset for all evoked and voluntary contractions was conducted manually using visual identification (Hannah et al. 2012, 2015), which is considered more valid than the use of automated identification methods (Tillin et al. 2013). Voluntary and evoked contractions were elicited in accordance with a previously published method (Hannah et al. 2012, 2015; Tillin et al. 2013) and are described below in brief.

\section{Resting twitches}

A single electrical impulse was delivered with stepwise increments in the current to evoke a twitch response, until a plateau in the amplitude of twitch force and compound muscle action potentials ( $\mathrm{M}$-waves) was reached. To ensure supramaximal stimulation, stimulus intensity was increased by $25 \%$ above the value required to evoke a plateau. Three discrete supramaximal stimuli were then evoked to elicit maximal twitch responses and M-waves. Electromechanical delay (EMD) was defined as the time difference between $\mathrm{M}$-wave onset (1st electrode site to be activated) and force onset. Twitch force at 25 and $50 \mathrm{~ms}$ from force onset, was measured as markers of the explosive force production, peak force, time-to-peak tension (TPT), and half-relaxation time (HRT) were also reported. All measurements were averaged across the three maximal twitch contractions. The M-wave response for the three quadriceps electrodes was measured for M-wave area, from EMG onset to the point where the signal returned to baseline, and averaged across the three sites. The mean M-wave area of the three supramaximal stimuli was defined as the maximal M-wave area $\left(M_{\max }\right)$ and was used for normalisation of voluntary quadriceps EMG.

\section{Maximum voluntary contractions and potentiated twitches}

Participants were instructed to produce four maximal voluntary isometric contractions (MVIC), "as hard as possible" for 3-4 s. Strong verbal encouragement reiterating the instructions was provided during and after each contraction, together with visual onscreen feedback. Following each MVIC, supramaximal stimulation of the femoral nerve at the same configuration and stimulus intensity as the resting twitches was elicited to evoke maximal potentiated twitch. MVIF was defined as the greatest instantaneous force during either the knee extensor MVICs or explosive voluntary contractions (see below). The root mean square (RMS) of the EMG signal for each muscle (RF, VM, and VL) was calculated over a $500 \mathrm{~ms}$ epoch surrounding MVIF (250 ms either side) and normalized to the corresponding $M_{\max }$. All sites were then averaged to calculate a mean quadriceps value. EMD, force at 25 and $50 \mathrm{~ms}$ from onset, peak twitch force, TPT, and HRT were averaged across the four maximal potentiated twitch contractions.

\section{Explosive voluntary contractions}

Participants completed isometric explosive voluntary contractions, starting each contraction completely relaxed, contracting their knee "as fast and hard as possible" for $\sim 1 \mathrm{~s}$, with an emphasis on "fast". The three contractions with the greatest maximum rate of force development, 
achieving the following criteria, were used for analysis: (1) no prior countermovement or pretension, and (2) peak force $>80 \%$ MVIF. Explosive force was measured at $25 \mathrm{~ms}$ intervals up to $150 \mathrm{~ms}$ after force onset. The RMS of the EMG signal from each muscle was measured over three consecutive $50 \mathrm{~ms}$ time periods from EMG onset of the first agonist muscle to be activated (i.e., 0-50, 50-100, and 100-150 ms). Thereafter, RMS at each EMG site was normalized to $M_{\max }$ and averaged to provide a mean quadriceps value. All measurements were averaged across the three selected contractions.

\section{Force-EMG relationship (via voluntary incremental knee extension contractions)}

Submaximal knee extensor contractions were completed at $15 \%$ increments of MVIF, in ascending order, separated by $\geq 20$ s. Force target levels were displayed on screen by horizontal cursors, with participants instructed to reach the target as quickly as possible, and then maintain this target force level as accurately as possible for $\sim 3 \mathrm{~s}$. During each contraction intensity, the RMS of the EMG and average force over a stable $500 \mathrm{~ms}$ part of the force trace (minimal standard deviation of the force trace for that contraction). The EMG RMS values were normalized to $M_{\max }$ and plotted against the respective force values. Linear regression was used to evaluate the slope and intercept of the force-EMG relationship incorporating all data between 15 and $90 \%$ MVIF.

\section{Octet contractions}

Octet contractions (8 impulses at $300 \mathrm{~Hz}$ ) were evoked via supramaximal stimulation of the femoral nerve. In summary, three discrete pulses ( $\geq 15 \mathrm{~s}$ apart) were delivered with a supramaximal current $(+25 \%)$ to evoke maximal octet contractions. The octet force response was measured at 25 and $50 \mathrm{~ms}$ from force onset, as well as at the peak. All measurements were averaged across the three analysed contractions.

\section{Force-frequency relationship}

Tetanic contractions were elicited via submaximal percutaneous electrical stimulation of the quadricep to examine the force-frequency relationship (Lamont and Miller 1992). $100 \mathrm{~Hz}$ contractions were evoked at increasing current intensities, $\geq 30 \mathrm{~s}$ apart, to determine the current that elicited $50 \%$ of MVIF. This current was then used for the following force-frequency measurements. The final calibration contraction at $100 \mathrm{~Hz}$ and the subsequent measured contractions were separated by $\geq 60 \mathrm{~s}$. The force-frequency relationship contractions consisted of two twitch contractions $(1 \mathrm{~Hz})$, followed by single contractions of $1 \mathrm{~s}$ duration at each of nine different frequencies $(5,10,15,20$, $30,40,50,80$, and $100 \mathrm{~Hz}$ ) performed in ascending order with $\geq 30$ s between contractions. Peak force was defined as the greatest instantaneous force. Thereafter, the force values at each stimulation frequency were normalized to the force obtained at $100 \mathrm{~Hz}$. The force-frequency relationship was fitted with a Hill curve and evaluated for frequency at $50 \%$ of the maximum force response (Dutka and Lamb 2004).

\section{Sustained isometric knee extensor hold}

To induce $\mathrm{H}^{+}$accumulation within the quadricep muscles, participants were instructed to perform a voluntary isometric contraction at $45 \%$ of MVIF for "as long as possible". The start of the sustained fatigue hold was defined as the time when force was greater than $40 \%$ of MVIF, and terminated when force fell below $5 \%$ of the target force for more than $3 \mathrm{~s}$, despite strong encouragement. Strong verbal encouragement was provided alongside visual feedback displayed onscreen. The time between start and end of the sustained fatigue was defined as the time-to-task failure (TTF), with average force recorded across this time. Impulse (kN.s) was calculated as the product of the average force and TTF. It has been estimated that TTF would be $\sim 78 \mathrm{~s}$ for a contraction held at $45 \%$ of MVIC force (Ahlborg et al. 1972). Immediately upon completion of the sustained fatigue hold, participants repeated all voluntary and evoked contractions.

\section{Blood samples}

Fingertip capillary blood samples were taken at rest, immediately prior to and $5 \mathrm{~min}$ following the sustained fatigue hold. Fingertip capillary blood lactate measured 5 min post-exercise provides an estimate of lower limb blood lactate contractions (Comeau et al. 2011). Sampling involved the collection of $80 \mu \mathrm{L}$ of whole blood into a heparincoated clinitube; all samples were analysed immediately post-sampling (Radiometer Ltd, UK).

\section{Statistical analysis}

Based on an a priori power calculation, a minimum of 22 participants were required to achieve $92 \%$ power at $P<0.05$. Calculations were based on the previous findings (Hobson et al. 2012), with 24 participants being recruited to allow for dropouts. Statistical analyses were completed using SPSS version 22 (SPSS Inc., Chicago, IL, USA), with statistical significance accepted at $P \leq 0.05$. Data are presented as means \pm 1 standard deviation (SD). Dependent variables (MVIF, EMD, HRT, TPT, slope, and intercept 
of force-EMG relationship, frequency at $50 \%$ of force response for the force-frequency relationship) were evaluated using a two-way mixed-model (group $\times$ session) analysis of variance (ANOVA). Dependent variables measured over several time points (force and EMG during explosive voluntary contractions, evoked twitch, and octet force) were analysed using a three-way mixed-model (group $\times$ session $\times$ time) ANOVA. All variables were assessed during both fresh and fatigued conditions. The sustained fatigue time-to-task failure (TTF) and impulse were analysed using a two-way mixed-model ANOVA.

The impact of the fatigue hold contraction on dependant variables (MVIF, EMD, HRT, TPT, slope, and intercept of force-EMG relationship, frequency at $50 \%$ of force response for the force-frequency relationship) was analysed using a three-way mixed-model (fatigue $\times$ group $\times$ session) ANOVA. Percentage change between fresh and fatigued values for the dependent variables measured over several time points (force and EMG during explosive voluntary contractions, evoked twitch, and octet force) were analysed using a three-way mixed-model (percentage change $\times$ group $\times$ session) ANOVA. A Greenhouse-Geisser correction was applied when the ANOVA assumption of sphericity was violated. Effect size for multiple comparisons was calculated using partial $\left(\eta_{p}^{2}\right)$ and generalised $\left(\eta_{g}^{2}\right)$ eta squared (Lakens 2013). Providing two effect sizes is suggested to yield a greater understanding of a specific effect (Preacher and Kelly 2011). Post hoc comparisons to explain any significant interactions are reported with Cohen's $d$ effect size. An effect size of $0.2-0.5$ was defined as small, $0.5-0.8$ as medium, and $\geq 0.8$ as large (Schünemann et al. 2008). Intra-individual variability was assessed using the mean intra-individual coefficient of variation (CV) across the two measurement sessions for the PLA group $[(\mathrm{SD} /$ mean $) \times 100]$, the current research $\mathrm{CVs}$ are in line with those reported previously using the same equipment (Hannah et al. 2015).

\section{Results}

\section{Electrically evoked contractile properties}

\section{Resting twitches}

Supplementation did not significantly influence twitch force, EMD, or TPT (Table 1). There was, however, a significant group $\times$ session interaction for HRT in both fresh $\left(P=0.04, n_{\mathrm{p}}^{2}=0.2, n_{\mathrm{g}}^{2}<0.001\right)$ and fatigued $\left(P=0.03, \eta_{\mathrm{p}}^{2}=\right.$ $0.2, \eta_{\mathrm{g}}^{2}=0.1$; Figs. 1,2) muscle. Post hoc analysis showed that the percentage change in fresh muscle HRT was not significantly different between the BA $(-2 \pm 10 \mathrm{~ms} ;-3 \pm$ $13 \%)$ and PLA group $(+8 \pm 16 \mathrm{~ms} ; 8 \pm 16 \%)$ with a large effect reported $(\mathrm{P}=0.06$; Cohen's $\mathrm{d}=0.9)$. In fatigued muscle, post hoc analysis showed that HRT percentage change was significantly different between the BA $(-25 \pm$ $34 \mathrm{~ms} ;-19 \pm 26 \%)$ and PLA $(8 \pm 16 \mathrm{~ms} ; 0 \pm 15 \%)$ group with a large effect reported ( $P=0.05$, Cohen's $d=0.9)$.

Table 1 Electrically femoral nerve evoked force responses, time-to-peak tension (TPT), and electromechanical delay (EMD) of $\beta$-alanine (BA) and placebo (PLA) groups pre- and post-supplementation, in fresh and fatigued muscle. Data are means $\pm 1 \mathrm{SD}$

\begin{tabular}{|c|c|c|c|c|c|c|c|c|c|c|c|}
\hline & & \multicolumn{5}{|c|}{ Pre-supplementation } & \multicolumn{5}{|c|}{ Post-supplementation } \\
\hline & & \multicolumn{3}{|c|}{ Force $(\mathrm{N})$} & \multirow[t]{2}{*}{ EMD (ms) } & \multirow[t]{2}{*}{ TPT (ms) } & \multicolumn{3}{|c|}{ Force $(\mathrm{N})$} & \multirow[t]{2}{*}{ EMD (ms) } & \multirow[t]{2}{*}{ TPT (ms) } \\
\hline & & 25 & 50 & Peak & & & 25 & 50 & Peak & & \\
\hline \multicolumn{12}{|c|}{ Resting twitch } \\
\hline Fresh & $\mathrm{BA}$ & $23 \pm 5$ & $73 \pm 14$ & $88 \pm 17$ & $10 \pm 1$ & $79 \pm 11$ & $24 \pm 6$ & $73 \pm 17$ & $90 \pm 19$ & $10 \pm 1$ & $81 \pm 9$ \\
\hline Fresh & PLA & $28 \pm 4$ & $79 \pm 19$ & $99 \pm 26$ & $11 \pm 1$ & $82 \pm 8$ & $29 \pm 5$ & $82 \pm 16$ & $104 \pm 24$ & $10 \pm 1$ & $83 \pm 9$ \\
\hline Fatigued & BA & $22 \pm 7$ & $61 \pm 22$ & $68 \pm 25$ & $11 \pm 2$ & $72 \pm 16$ & $26 \pm 8$ & $71 \pm 22$ & $79 \pm 23$ & $10 \pm 1$ & $74 \pm 13$ \\
\hline Fatigued & PLA & $25 \pm 6$ & $58 \pm 20$ & $65 \pm 24$ & $11 \pm 1$ & $69 \pm 8$ & $24 \pm 4$ & $60 \pm 15$ & $67 \pm 18$ & $10 \pm 2$ & $71 \pm 9$ \\
\hline \multicolumn{12}{|c|}{ Potentiated twitch } \\
\hline Fresh & BA & $55 \pm 13$ & $142 \pm 24$ & $159 \pm 26$ & $9 \pm 1$ & $79 \pm 6$ & $53 \pm 8$ & $136 \pm 14$ & $155 \pm 9$ & $9 \pm 1$ & $81 \pm 6$ \\
\hline Fresh & PLA & $60 \pm 16$ & $138 \pm 37$ & $163 \pm 43$ & $9 \pm 1$ & $79 \pm 9$ & $59 \pm 10$ & $142 \pm 34$ & $167 \pm 38$ & $9 \pm 1$ & $82 \pm 8$ \\
\hline Fatigued & BA & $37 \pm 11$ & $92 \pm 27$ & $101 \pm 29$ & $11 \pm 1$ & $74 \pm 9$ & $40 \pm 9$ & $101 \pm 19$ & $110 \pm 18$ & $10 \pm 1$ & $73 \pm 10$ \\
\hline Fatigued & PLA & $42 \pm 10$ & $93 \pm 25$ & $105 \pm 30$ & $10 \pm 1$ & $74 \pm 4$ & $38 \pm 9$ & $93 \pm 23$ & $106 \pm 28$ & $10 \pm 1$ & $74 \pm 8$ \\
\hline \multicolumn{12}{|l|}{ Octet } \\
\hline Fresh & BA & $52 \pm 15$ & $165 \pm 42$ & $223 \pm 47$ & $7 \pm 2$ & $117 \pm 25$ & $64 \pm 18$ & $196 \pm 37$ & $274 \pm 53$ & $7 \pm 2$ & $120 \pm 24$ \\
\hline Fresh & PLA & $66 \pm 17$ & $190 \pm 62$ & $285 \pm 94$ & $6 \pm 1$ & $127 \pm 19$ & $64 \pm 17$ & $199 \pm 60$ & $296 \pm 94$ & $7 \pm 2$ & $137 \pm 7$ \\
\hline Fatigued & BA & $62 \pm 23$ & $191 \pm 49$ & $221 \pm 72$ & $7 \pm 2$ & $100 \pm 23$ & $62 \pm 17$ & $182 \pm 47$ & $244 \pm 68$ & $7 \pm 2$ & $114 \pm 25$ \\
\hline Fatigued & PLA & $69 \pm 20$ & $191 \pm 49$ & $273 \pm 72$ & $7 \pm 1$ & $123 \pm 14$ & $64 \pm 16$ & $198 \pm 49$ & $283 \pm 79$ & $7 \pm 2$ & $125 \pm 15$ \\
\hline
\end{tabular}


Fig. 1 Electrically evoked half-relaxation time of $\beta$-alanine (BA) and placebo (PLA) groups pre- and postsupplementation, in fresh and fatigued muscle during: resting twitch (a), potentiated twitch (b), and octets (c). Data are means $\pm 1 \mathrm{SD}$. $* * P \leq 0.01$ and $* P \leq 0.05$ for post hoc independent $t$-test between BA and PLA groups
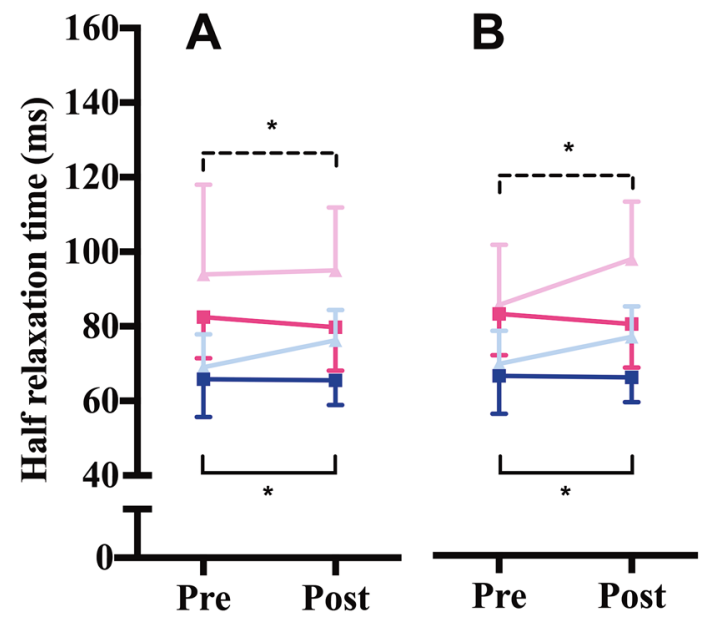

$\because$ BA fresh

PLA fresh

BA fatigued

PLA fatigued

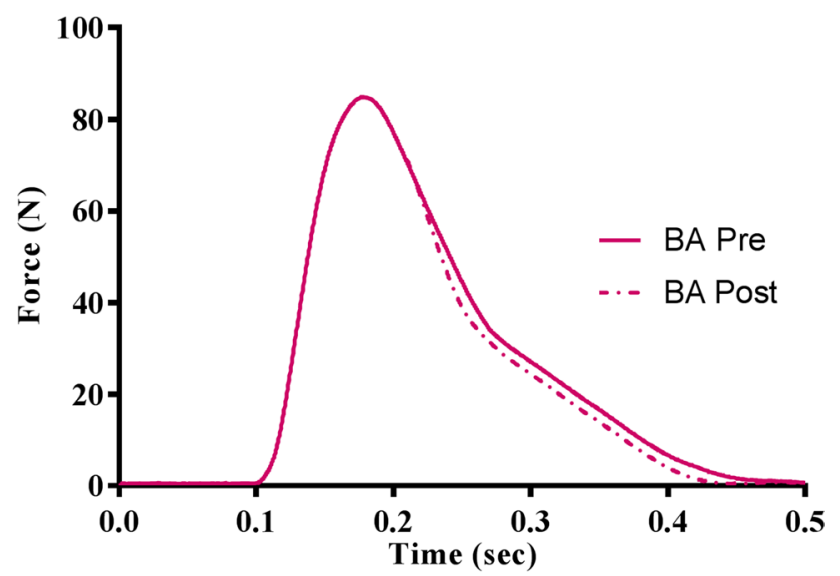

Fig. 2 Representative records of the force response during an electrically evoked resting twitch contraction pre- and post-supplementation with $\beta$-alanine under fresh conditions. These records are averaged records from three participants to provide an illustration of the decline in twitch half-relaxation time

The percentage difference between fresh and fatigued resting twitch force remained similar between sessions for both supplementation groups. Resting twitch TPT declined following completion of the sustained fatigue hold $\left(P=0.001, \eta_{p}^{2} \eta_{\mathrm{p}}^{2}=0.4, \eta_{g}^{2}=0.2\right)$, although EMD remained similar with no difference between sessions or groups (Table 1). Resting twitch HRT significantly increased following the completion of the sustained fatigue hold $(P$ $<0.001, n_{p}^{2}=0.7, n_{g}^{2}=0.3$; Fig. 1$)$ with no group $\times$ fatigue or group $\times$ session $\times$ fatigue interactions.

\section{Potentiated twitches}

Supplementation did not significantly influence twitch force, EMD or TPT (Table 1). There was, however, a significant group $\times$ session interaction for HRT in both fresh
$\left(P=0.03, n_{p}^{2}=0.2, n_{g}^{2}<0.001\right)$ and fatigued muscle $\left(P=0.03, \eta_{p}^{2}=0.2, \eta_{g}^{2}<0.001\right.$; Figs. 1,2$)$. Post hoc analysis showed that the percentage change in fresh muscle HRT was not significantly different between the BA $(0 \pm 9 \mathrm{~ms}$; $+1 \pm 15 \%)$ and PLA group $(+7 \pm 10 \mathrm{~ms} ;+12 \pm 15 \%)$ with a medium effect reported $(P=0.10$, Cohen's $d=0.7)$. In fatigued muscle, post hoc analysis showed that the percentage change in fatigued muscle HRT was significantly different between the BA $(-2.7 \pm 16 \mathrm{~ms} ;-2 \pm 20 \%)$ and PLA group $(12 \pm 12 \mathrm{~ms} ; 16 \pm 17 \%)$ with a large effect reported $(P=0.03$, Cohen's $d=1.0)$.

The percentage difference between fresh and fatigued potentiated twitch force remained similar between sessions for both supplementation groups, with only a significant effect of time $\left(P=0.001, \eta_{p}^{2}=0.5, \eta_{g}^{2}=0.5\right)$. Potentiated twitch TPT declined following completion of the sustained fatigue hold $\left(P<0.001, \eta_{p}^{2}=0.5, \eta_{g}^{2}=0.5\right.$; Table 1). Potentiated EMD significantly prolonged following the fatigue hold $\left(P=0.001, \eta_{p}^{2}=0.45, \eta_{g}^{2}=0.2\right)$ with no group $\times$ fatigue or group $\times$ session $\times$ fatigue interactions. Potentiated HRT significantly increased following the completion of the sustained fatigue hold $\left(P<0.001, \eta_{p}^{2}=0.7\right.$, $\eta_{g}^{2}=0.4$; Fig. 1), with no group $\times$ fatigue or group $\times$ session $\times$ fatigue interactions.

\section{Octet contractions}

In both fresh and fatigued muscle, supplementation did not significantly alter octet peak force, EMD or TPT (Table 1). There was, however, a significant group $\times$ session interaction for octet HRT in both fresh $\left(P=0.05 ; n_{p}^{2}=0.2 ; \eta_{g}^{2}=\right.$ $0.1)$ and fatigued $\left(P=0.01 ; n_{p}^{2}=0.3 ; \eta_{g}^{2}=0.2\right.$; Figs. 1 , 2) skeletal muscle. Post hoc analysis showed that the percentage change in fresh muscle was significantly different between the BA $(-26 \pm 30 \mathrm{~ms} ;-20 \pm 22 \%)$ and PLA $(0 \pm 32 \mathrm{~ms} ; 1 \pm 34 \%)$ group with a large effect reported 
( $P=0.05$, Cohen's $d=0.8)$. In fatigued muscle, post hoc analysis showed that HRT percentage change was significantly different between the BA $(-11 \pm 20 \mathrm{~ms} ;-11 \pm$ $20 \%)$ and PLA $(12 \pm 19 \mathrm{~ms} ; 7 \pm 13 \%)$ groups with a large effect reported ( $P=0.01$, Cohen's $d=1.2)$.

Octet force percentage change between fresh and fatigue was not significantly affected by supplementation. Octet TPT $\left(P=0.05, \eta_{p}^{2}=0.2, \eta_{g}^{2}=0.1\right.$; Table 1) and HRT ( $P=0.008, \eta_{p}^{2}=0.3, \eta_{g}^{2}=0.2$; Fig. 1$)$, declined following completion of the sustained fatigue hold with no group $\times$ fatigue or group $\times$ session $\times$ fatigue interactions. Octet EMD was not significantly influenced following the fatigue hold, with no group $\times$ fatigue or group $\times$ session $\times$ fatigue interactions (Table 1 ).

\section{Force-frequency relationship}

Supplementation did not significantly influence peak force at each frequency of stimulation, and the frequency at 50\% of the force response (Table 2) in either fresh or fatigued muscle. Following the fatigue hold, peak force significantly declined $\left(P=0.001, \eta_{p}^{2}=0.8, \eta_{g}^{2}=0.2\right)$, although the frequency at $50 \%$ of the force response remained unaffected.

\section{Maximum and explosive voluntary force production}

Supplementation had no effect on MVIF in fresh or fatigued muscle (Fig. 3a). Following the fatigue hold, MVIC significantly declined $\left(P<0.001, \eta_{p}^{2}=0.9, \eta_{g}^{2}=0.2\right)$, with no differences between session and groups (BA: 17-18\%; PLA: $21 \%$; Fig. 3a). There was no effect of supplementation on force measures at $25 \mathrm{~ms}$ intervals during explosive voluntary contractions in fresh and fatigued states (Fig. 3a). Explosive force percentage change between fresh and fatigue of force remained unaffected by supplementation.

\section{Neuromuscular activation}

\section{Agonist neuromuscular activation during maximal and explosive voluntary contractions}

Agonist EMG normalized to $M_{\max }$ during MVICs and explosive contraction remained uninfluenced by supplementation in fresh and fatigued muscles (Fig. 3b).

\section{Force-EMG relationship}

The slope and y-intercept of the force-EMG relationship were unaffected by supplementation in both fresh and fatigued muscles (Fig. 4; Table 2). The percentage change in agonist EMG normalized to $M_{\max }$ during MVICs and explosive contraction was not significantly altered between sessions.

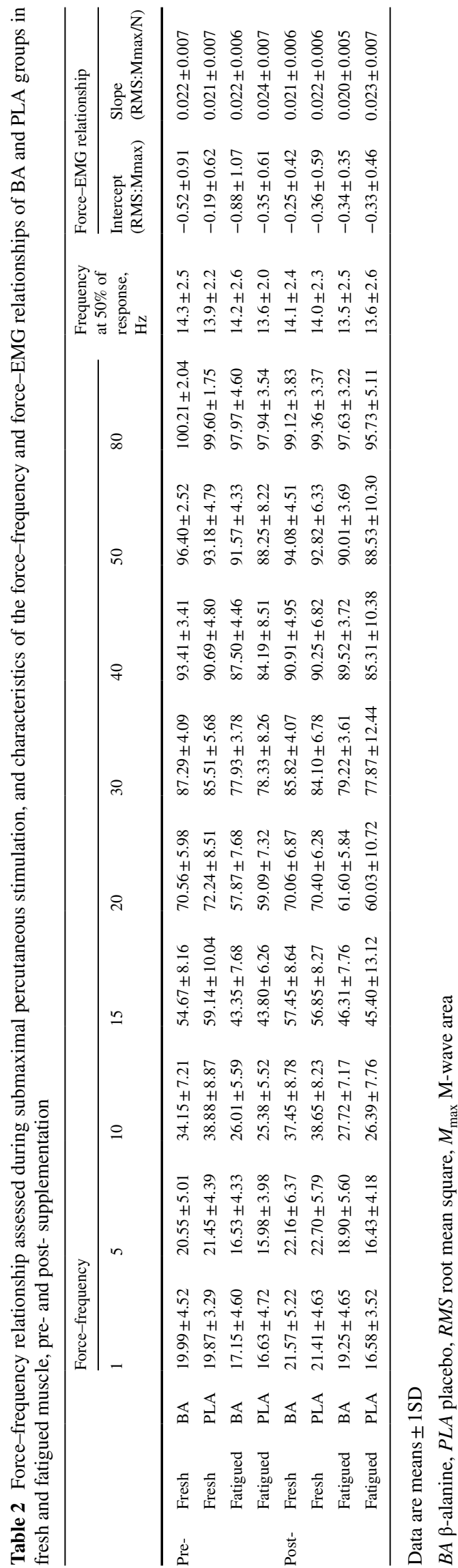



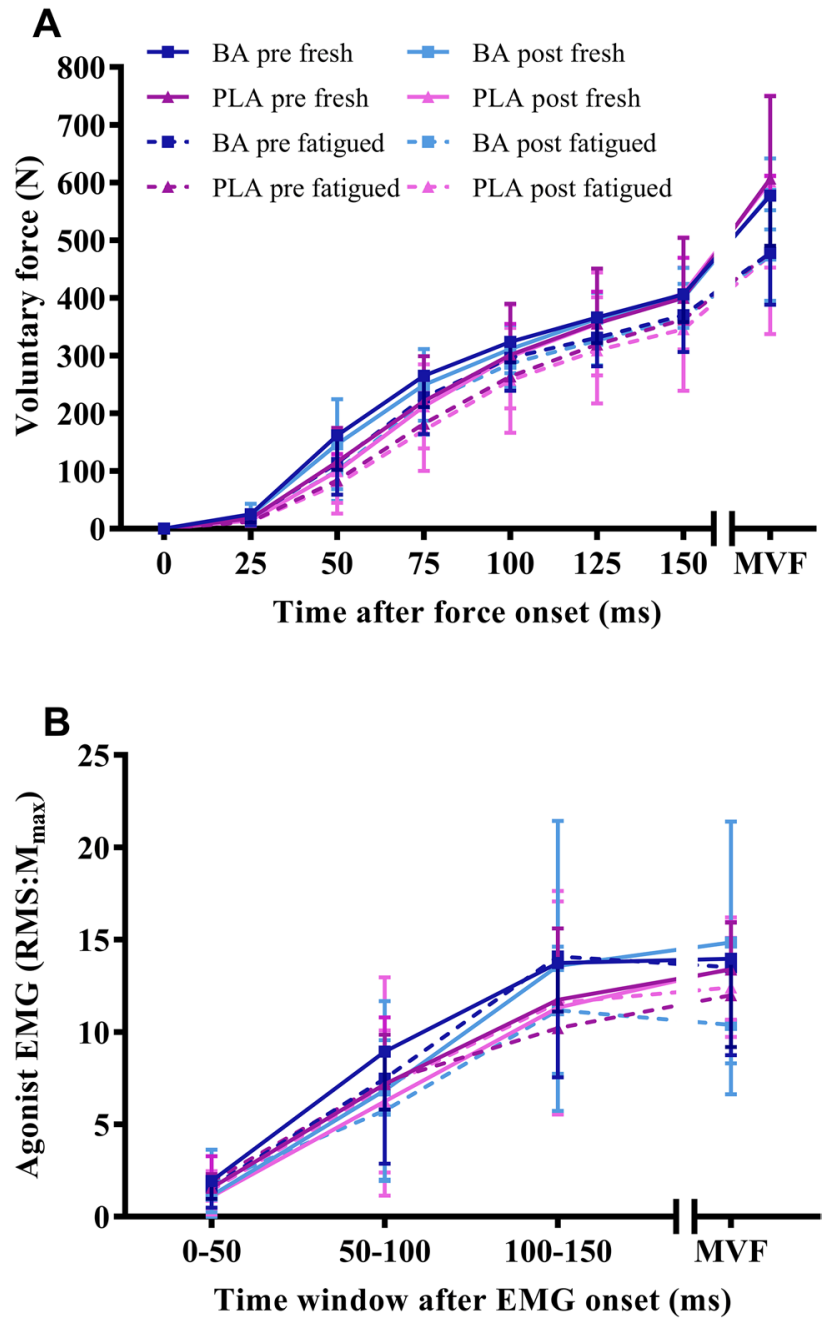

Fig. 3 Explosive and maximal voluntary isometric force (MVIF) responses (a), and agonist EMG normalized to $\mathrm{M}$-wave area $\left(\mathrm{M}_{\max }\right)$ during explosive contractions $(0-50,50-100$, and $100-150 \mathrm{~ms}$ from onset) and at MVIF (b) for the BA and PLA groups pre- and post-supplementation, in fresh and fatigued muscles. Data are means $\pm 1 \mathrm{SD}$

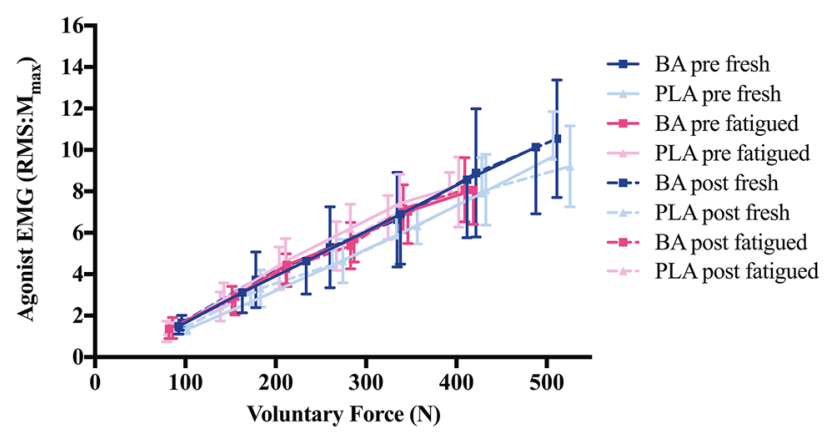

Fig. 4 Force-EMG relationship measured during submaximal voluntary contractions (15-90\% MVIF) for the BA and PLA groups preand post-supplementation, in fresh and fatigued muscles. Data are means $\pm 1 \mathrm{SD}$

\section{Sustained isometric knee extensor hold}

TTF was unaffected by BA (pre: $63.2 \pm 13.0 \mathrm{~s}$; post: $63.4 \pm 15.3 \mathrm{~s}$ ) or PLA supplementation (pre: $77.3 \pm 24.8 \mathrm{~s}$; Post: $75.3 \pm 18.9$ s). Impulse was also not significantly influenced by BA (pre: $16.1 \pm 3.5 \mathrm{kN} \mathrm{s}^{-1}$; post $16.4 \pm 4.6$ $\mathrm{kN} \mathrm{s}^{-1}$ ) or PLA supplementation (pre: $19.6 \pm 4.4 \mathrm{kN} \mathrm{s}^{-1}$; post $19.4 \pm 3.2 \mathrm{kN} \mathrm{s}^{-1}$ ). Blood lactate concentrations at rest and prior to the sustained fatigued hold were not significantly different (Table 3). Blood lactate concentrations significantly increased $5 \mathrm{~min}$ following the sustained isometric knee extensor hold compared to both rest and prior to values $\left(P<0.001, \eta_{p}^{2}=0.8, \eta_{g}^{2}=0.6\right)$, with no difference between sessions or group (Table 3 ).

\section{Discussion}

The key findings from the present study are: (a) no effects of BA supplementation on isometric force production capacity in either fresh or fatigued skeletal muscle, (b) the confirmation of our previous findings (Hannah et al. 2015) showing altered fresh muscle relaxation speed following 28 days of BA supplementation, and (c) that the skeletal muscle relaxation speed is also reduced by BA supplementation following muscle fatigue in the absence of any change to peak force production or contraction time compared to the PLA group. The current investigation examined the influence of BA supplementation on neuromuscular performance measures, associated with $\mathrm{Ca}^{2+}$ handling within the skeletal muscle, a proposed mechanism associated with the improvements in exercise performance (Dutka and Lamb 2004; Everaert et al. 2013; Guglielmi et al. 2013; Sale et al. 2013) in fresh and fatigued muscle conditions. These data are the first to comprehensively examine the effect of BA supplementation on voluntary and electrically evoked contractile properties of in-vivo fatigued human skeletal muscle.

During both fresh and fatigued conditions, BA supplementation has no effect on voluntary isometric force production including maximal and explosive force variables. Voluntary force peak data are consistent with the lack of change in electrically evoked peak force responses noted during twitch and octet contractions under both fresh and fatigued conditions. There were similar neural drive responses during both MVICs and explosive contractions pre- and post-supplementation in fresh and fatigued muscle. The current findings in fresh muscle are in-line with the previous findings (Hannah et al. 2015), it was proposed that if BA supplementation had influenced $\mathrm{Ca}^{2+}$ related function, improved explosive voluntary force and/or alterations in the force-EMG relationship would have been evident. 
Table 3 Blood lactate concentrations $\left(\mathrm{mmol} \cdot \mathrm{l}^{-1}\right.$ ) for the BA and PLA groups pre- and post-supplementation, at rest, prior to, and 5 min following the completion of the sustained fatigue hold $(+5 \mathrm{~min})$

\begin{tabular}{llllllll}
\hline & \multicolumn{2}{l}{ Pre-supplementation } & & \multicolumn{2}{l}{ Post-supplementation } \\
\cline { 2 - 3 } & Rest $\left(\mathrm{mmol} \cdot \mathrm{l}^{-1}\right)$ & Prior to $\left(\mathrm{mmol} \cdot \mathrm{l}^{-1}\right)$ & $+5 \min \left(\mathrm{mmol} \cdot \mathrm{l}^{-1}\right)$ & & Rest $\left(\mathrm{mmol} \cdot \mathrm{l}^{-1}\right)$ & Prior to $\left(\mathrm{mmol} \cdot \mathrm{l}^{-1}\right)$ & $+5 \mathrm{~min}\left(\mathrm{mmol} \cdot \mathrm{l}^{-1}\right)$ \\
\hline BA & $1.0 \pm 0.3$ & $1.1 \pm 0.2$ & $3.8 \pm 1.1^{*} \mathrm{X}$ & & $1.2 \pm 0.3$ & $1.3 \pm 0.4$ & $3.7 \pm 1.3^{*} \mathrm{X}$ \\
PLA & $1.0 \pm 0.3$ & $1.1 \pm 0.4$ & $3.8 \pm 1.3^{*} \mathrm{X}$ & & $1.3 \pm 0.3$ & $1.2 \pm 0.2$ & $4.2 \pm 1.0^{*} \mathrm{X}$ \\
\hline
\end{tabular}

Significant differences between concentrations are denoted by * (rest and $+5 \mathrm{~min}$ ) and ${ }^{\mathrm{X}}$ (prior to and $+5 \mathrm{~min}$ ). Data are means $\pm 1 \mathrm{SD}$

In both fresh and fatigued in-vivo human skeletal muscles, there was no leftward shift of the force-frequency curve, the associated measure of intracellular $\mathrm{Ca}^{2+}$ levels (Batrukova and Rubstov 1997). Thus suggesting that elevation of carnosine concentrations did not significantly alter $\mathrm{Ca}^{2+}$-related function. That said, there may have been a decline in sarcoplasmic reticulum $\mathrm{Ca}^{2+}$ release which was not documented in the force-frequency curve, due to an associated increase in $\mathrm{Ca}^{2+}$ sensitivity of the myofibrils, resulting in the same skeletal muscle force. The current research in fresh muscle is in line with the previous in-vivo research (Hannah et al. 2015), where increased muscle carnosine concentration following a similar 28-day BA supplementation protocol did not alter the force-frequency curve. One potential limitation of both studies is that neither measured intracellular carnosine concentrations directly. That said there are many studies displaying increased muscle carnosine following BA supplementation, almost without exception, on an individual-by-individual basis (Harris et al. 2006; Hill et al. 2007). Thus, we are confident in assuming that a significant increase in muscle carnosine content would have occurred with the BA supplementation protocol implemented in the present study.

The lack of an effect of BA on TPT and force production following increased carnosine concentrations is interesting. Based on the previous in-vitro studies in chemically skinned muscle fibres from frogs (Lamont and Miller 1992), mechanically skinned rat muscle fibres (Dutka and Lamb 2004) and type I and type II human skeletal muscle fibres (Dutka et al. 2012), an alteration to submaximal action potential-mediated force responses via increased $\mathrm{Ca}^{2+}$ sensitivity could have been expected. Furthermore, the current investigation reported no alteration to potentiated twitch contractions. Given that the phosphorylation of the myosin head during these twitches is impacted by $\mathrm{Ca}^{2+}$ sensitivity, any impact of increased carnosine concentrations would have been displayed as a resultant effect on these measures. In-vitro data following BA supplementation in mice reported increased carnosine $(+156 \%)$ and anserine content $(+46 \%)$ in the extensor digitorum longus muscle and a marked leftward shift of the force-frequency relationship (Everaert et al. 2013). These alterations to skinned muscle fibre $\mathrm{Ca}^{2+}$ handling when exposed to increased carnosine concentrations are interesting, although both the previous (Hannah et al. 2015) and current in-vivo studies suggest that these responses might not be significant enough to be evident during whole muscle contraction. It might be that the differences between these in-vitro data (where carnosine can be indirectly elevated to a consistent level) and the data from the current study reflect differences in the magnitude of intramuscular carnosine elevation, which we, unfortunately, cannot confirm in the current study. It is also important to note that in-vitro research is conducted outside the normal intracellular environment, and importantly, a number of protocols include the use of free magnesium, an inhibitor of skeletal muscle ryanodine receptors (Laver et al. 2004). Furthermore, during in-vitro studies solutions are added to control $\mathrm{pH}$ levels allowing examination of the direct effect of carnosine, although this is important, these investigations have yet to examine the influence of carnosine concentration and varying $\mathrm{pH}$ levels. The current body of in-vivo research is completely separate from the in-vitro data, which might make it unrealistic to expect similar findings between research designs.

Increasing muscle carnosine concentrations with 28 days of BA supplementation resulted in a shorter HRT (relative to equivalent PLA times) in fresh and fatigued skeletal muscle during both resting and potentiated twitch contractions. These are in contrast with HRT in fresh and fatigued resting and potentiated twitch contractions following PLA supplementation. The altered muscle HRT values in the current investigation are in-line with those previously reported following the same BA supplementation protocol (7-12\%; Hannah et al. 2015). Muscle relaxation speed has been associated with both $\mathrm{Ca}^{2+}$ removal from the myoplasm $\left(\mathrm{Ca}^{2+}\right.$ component) and $\mathrm{Ca}^{2+}$ dissociation from troponin followed by cross-bridge detachment (cross-bridge component) (Westerblad et al. 1997). Research conducted by Westerblad and Allen (1993), in fatigued mouse muscle fibres, suggested that the slowing of muscle relaxation, apparent under fatigued conditions, was a reflection of slowed cross-bridge kinetics, rather than a reduction in the rate of $\mathrm{Ca}^{2+}$ decline at the end of the stimulation train. Yet, when these data were repeated in Xenopus muscle fibres, the slowing of muscle relaxation was associated with a combination of altered cross-bridge kinetics and impaired 
$\mathrm{Ca}^{2+}$ handling, rather than just slowed cross-bridge kinetics alone. As such, the decline in skeletal muscle HRT following 28 days of BA supplementation shown in the current investigation may be associated with alterations to skeletal muscle cross-bridge kinetics. Alternatively, the decrease in HRT may be associated with the $\mathrm{Ca}^{2+}$ component of skeletal muscle relaxation speed, given that it has been proposed that $\mathrm{Ca}^{2+}$ re-uptake by the sarcoplasmic reticulum $\mathrm{Ca}^{2+}$-ATPase (SERCA) is the rate-limiting step in muscle relaxation (Gillis 1985; Dux 1993). The transfer of $\mathrm{Ca}^{2+}$ into the SR lumen by the SERCA pump is accompanied by a counter-transport of $\mathrm{H}^{+}$out into the cytosol (Tran et al. 2009), by acting on $\mathrm{Ca}^{2+}$-handling proteins directly or via other molecules, $\mathrm{Ca}^{2+}$ signalling can be inhibited or excited (Swietach et al. 2013). The presence of carnosine has already been shown to improve isolated rat heart muscle contraction and increases free intracellular $\mathrm{Ca}^{2+}$ concentrations (Zaloga et al. 1996). At a pH of 6.0, where a complete decline in $\mathrm{Ca}^{2+}$ release pump activity was evident, the presence of carnosine maintained $30 \%$ of pump activity at the same $\mathrm{pH}$. Although speculative, these data suggest that increasing carnosine concentrations might alter $\mathrm{Ca}^{2+}$-channel activity by interacting with the $\mathrm{Ca}^{2+}$-channel itself (Batrukova and Rubstov 1997), possibly via the existence of saturable binding site(s) for carnosine on the $\mathrm{Ca}^{2+}$-channel. These data are, however, limited by a number of methodological factors, including but not limited to, the lack of a $\mathrm{Ca}^{2+}$ buffer, the overloading of the sarcoplasmic reticulum with $\mathrm{Ca}^{2+}$ concentrations approximately ten times greater than normal, and the addition of un-physiological magnesium concentrations. As such, care needs to be taken over the interpretation of these findings. Alternatively, the decrease in HRT may be mediated through improved $\mathrm{pH}$ control in the microenvironment of the $\mathrm{Ca}^{2+}$ release pump where rapid ATP hydrolysis will result in increased release of protons. There could also be an indirect mechanism to explain the beneficial effects displayed within the current investigation in regards to muscle relaxation. Given the number of other proteins that bind to $\mathrm{Ca}^{2+}$-channels (Berchtold et al. 2000), carnosine may alter the protein interactions with the $\mathrm{Ca}^{2+}$-channels and/ or bind with the proteins themselves; both of these mechanisms could influence the activity of the $\mathrm{Ca}^{2+}$-channel via increased carnosine concentrations.

Although the mechanism for reducing skeletal muscle relaxation time following BA supplementation remains unclear, such an outcome might be beneficial to exercise performance, especially during short, repeated muscle contractions where muscle relaxation comprises an important proportion of total energy consumption (Bergstrom and Hultman 1988). During concentric contractions, improvement of muscle recovery time has been shown to be critical to the amount of post-shortening force decrease (Edman
1975). Reducing relaxation rates may improve muscle power output and exercise performance. These findings are particularly important for activities where fast, repetitive contractions, and relaxations occur with no period of rest. Future research is essential to confirm an effect of BA supplementation and/or muscle carnosine accumulation on SERCA activity and to better understand how these isolated muscle effects might relate to repetitive sporting movements and overall performance. It would be of benefit to repeat these data in elite athletes where small changes to HRT might be advantageous. Equally we might speculate that benefits may occur in clinical populations such as Brody disease (where SERCA1 activity is significantly reduced; Guglielmi et al. 2013) or Duchenne Muscular Dystrophy (where an excess of cytosol $\mathrm{Ca}^{2+}$ occurs; Ohlendieck 2000).

Within the current investigation, isometric knee extensor fatigue hold times were not significantly influenced by BA or PLA supplementation, in direct contrast to our previous findings that showed a $13.2 \%(9.7 \pm 9.4 \mathrm{~s})$ increase in $45 \%$ hold times following BA supplementation (Sale et al. 2012). The reason for a lack of a significant effect in the current study is unclear, given that isometric knee extensor hold times reported by both investigations were similar and aligned to times predicted by the Rohmert equation at a 45\% MVIC (78 s; Ahlborg et al. 1972). At 45\% MVIC, blood flow is occluded and thus the active muscle fibres are largely dependent upon anaerobic energy provision (Ahlborg et al. 1972). Blood lactate sampled from the finger 5 min post-exercise is indicative of the lower extremity lactate release (Comeau et al. 2011), with groups in the current study displaying similar levels of lactate accumulation in the lower limb. To greater understand the relationship between skeletal muscle HRT, increased carnosine concentrations and muscle fatigue, further investigations implementing a dynamic fatiguing protocol are required, since contractile slowing (i.e., prolonged half-relaxation time) would affect shortening velocity and power output (Jones et al. 2006).

\section{Conclusion}

The current investigation showed that 28 days of BA supplementation enhanced muscle relaxation time in both fresh and fatigued skeletal muscle. Whilst this finding is of interest, it remains unclear as to whether it would be sufficient to result in improved exercise performance, particularly in the absence of any changes to the force-frequency relationship, peak force production, or contraction time. The mechanism for the ergogenic effect on muscle relaxation following increased carnosine content remains unclear. It could however, be proposed that $\mathrm{Ca}^{2+}$ re-uptake 
via direct or indirect mechanisms associated with SERCA pump activity is involved, as this is the rate-limiting step of muscle relaxation.

Author contributions All experiments were performed at Nottingham Trent University (Department of Sport Science). The study was designed as part of a wider research project by RLJ, CTB, RH, WF and CS. Acquisition and analysis of data for the work by RLJ, JD, and $\mathrm{BM}$; data interpretation and manuscript preparation were undertaken by RLJ, CTB, JD, BM, WF, RH, and CS. All authors approved the final version of the paper. All authors agree to be accountable for all aspects of the work in ensuring that questions related to the accuracy or integrity of any part of the work are appropriately investigated and resolved. All persons designated as authors qualify for authorship, and all those who qualify for authorship are listed.

\section{Compliance with ethical standards}

Funding This study was funded by and completed at Nottingham Trent University. The $\beta$-alanine and maltodextrin supplements for this study were provided free of charge from Natural Alternatives International (San Marcos, California), although no additional funding was provided. Roger Harris is an independent paid consultant of NAI and is named as an inventor on patents held by NAI.

Open Access This article is distributed under the terms of the Creative Commons Attribution 4.0 International License (http:// creativecommons.org/licenses/by/4.0/), which permits unrestricted use, distribution, and reproduction in any medium, provided you give appropriate credit to the original author(s) and the source, provide a link to the Creative Commons license, and indicate if changes were made.

\section{References}

Ahlborg B, Bergstrom J, Ekelund LG et al (1972) Muscle metabolism during isometric exercise performed at constant force. J Appl Physiol 33:224

Allen DG, Lannergren J, Westerblad H (1995) Muscle cell function during prolonged activity: Cellular mechanisms of fatigue. Exp Physiol 80:497-527

Allen DG, Lamb GD, Westerblad H (2008) Skeletal muscle fatigue: Cellular mechanisms. Physiol Rev 88:287

Baran EJ (2000) Metal complexes of carnosine. Biochem 65:789

Batrukova MA, Rubtsov AM (1997) Histidine-containing dipeptides as endogenous regulators of the activity of sarcoplasmic reticulum Ca-release channels. Biochimica et Biophysica Acta Biomembranes 1324:142.

Berchtold MW, Brinkmeier H, Müntener M (2000) Calcium ion in skeletal muscle: its crucial role for muscle function plasticity and disease. Physiol Rev 80:1215

Bergström M, Hultman E (1988) Energy cost and fatigue during intermittent electrical stimulation of human skeletal muscle. J Appl Physiol 65:1500

Comeau MJ, Adams TM, Church JB, Graves MM, Lawson PM (2011) Prediction of lower extremity lactate levels in exercising muscle utilizing upper extremity sampling sites. JEP 14:20

Decombaz J, Beaumont M, Vuichoud J, Bouisset F, Stellingwerff T (2012) Effect of slow-release beta-alanine tablets on absorption kinetics and paresthesia. Amino Acids 43:67
Dutka TL, Lamb GD (2004) Effect of carnosine on excitation-contraction coupling in mechanically-skinned rat skeletal muscle. J Muscle Res Cell Motil 25:203

Dutka TL, Lamboley CR, McKenna MJ, Murphy RM, Lamb GD (2012) Effects of carnosine on contractile apparatus $\mathrm{Ca}^{2+}$ sensitivity and sarcoplasmic reticulum $\mathrm{Ca}(2)(+)$ release in human skeletal muscle fibers. J Appl Physiol 112:728

Dux L (1993) Muscle relaxation and sarcoplasmic reticulum function in different muscle types. Rev Physiol Biochem Pharmacol 122:69

Edman KA (1975) Mechanical deactivation induced by active shortening in isolated muscle fibres of the frog. J Physiol 246:255

Everaert I, Stegen S, Vanheel B, Taes Y, Derave W (2013) Effect of beta-alanine and carnosine supplementation on muscle contractility in mice. Med Sci Sports Exerc 45:43

Gillis JM (1985) Relaxation of vertebrate skeletal muscle: a synthesis of the biochemical and physiological approaches. Biochimica et Biophysica Acta - Reviews on Bioenergetics 811:97

Guglielmi V, Voermans NC, Gualndi F et al (2013) Fourty-four years of brody disease: it is time to review. Genetic Syndromes Gene Therapy 4:9

Hannah R, Minshull C, Buckthorpe MW, Folland JP (2012) Explosive neuromuscular performance of males versus females. Exp Physiol 97:618

Hannah R, Stannard RL, Minshull C, Artioli GG, Harris RC, Sale C (2015) $\beta$-Alanine supplementation enhances human skeletal muscle relaxation speed but not force production capacity. J Appl Physiol 118:604

Harris RC, Tallon MJ, Dunnett M et al (2006) The absorption of orally supplied beta-alanine and its effect on muscle carnosine synthesis in human vastus lateralis. Amino Acids 30:279

Hill CA, Harris RC, Kim HJ et al (2007) Influence of beta-alanine supplementation on skeletal muscle carnosine concentrations and high intensity cycling capacity. Amino Acids 32:225

Hobson RM, Saunders B, Ball G, Harris RC, Sale C (2012) Effects of beta-alanine supplementation on exercise performance: a metaanalysis. Amino Acids 43:25

Jones DA, de Ruiter CJ, de Haan A (2006) Change in contractile properties of human muscle in relationship to the loss of power and slowing of relaxation seen with fatigue. J Physiol 576:913

Lakens D (2013) Calculating and reporting effect sizes to facilitate cumulative science: a practical primer for $t$-tests and ANOVAs. Front Psychol 4:863

Lamont C, Miller DJ (1992) Calcium sensitizing action of carnosine and other endogenous imidazoles in chemically skinned striated muscle. J Physiol 454:421

Laver DR, Eager KR, Taoube L, Lamb GD (2000) Effects of cytoplasmic and luminal $\mathrm{pH}$ on $\mathrm{Ca}^{2+}$ release channels from rabbit skeletal muscle. Biophys J 78:1835

Laver DR, O'Neill ER, Lamb GD (2004) Luminal $\mathrm{Ca}^{2+}$-regulated $\mathrm{Mg}^{2+}$ inhibition of skeletal ryanodine receptors reconstituted as isolated channels or coupled clusters. J Gen Physiol 124:741

Little SC, Tikunova SB, Norman C, Swartz DR, Davis JP (2011) Measurement of calcium dissociation rates from troponin $\mathrm{C}$ in rigor skeletal myofibrils. Front Physiol 2:70

Muntener M, Kaser L, Weber J, Berchtold MW (1995) Increase of skeletal muscle relaxation speed by direct injection of parvalbumin cDNA. Proc Natl Acad Sci USA 92:6504

Nogueira L, Shiah AA, Gandra PG, Hogan MC (2013) Ca(2) (+)-pumping impairment during repetitive fatiguing contractions in single myofibers: role of cross-bridge cycling. Am J Physiol Regul Integr Comp Physiol 305:R118

Ohlendieck K (2000) The Pathophysiological Role of Impaired Calcium Handling in Muscular Dystrophy-Madame Curie Bioscience Database-NCBI Bookshelf" In Madame Curie Bioscience 
http://www.ncbinlmnihgov/books/NBK6173 [accessed 27th January 2016]

Preacher KJ, Kelley K (2011) Effect size measures for mediation models: quantitative strategies for communicating indirect effects. Psychol Methods 16:93

Sale C, Saunders B, Harris RC (2010) Effect of beta-alanine supplementation on muscle carnosine concentrations and exercise performance. Amino Acids 39:321

Sale C, Hill CA, Ponte J, Harris RC (2012) Beta-Alanine Supplementation Improves Isometric Endurance of the Knee Extensor Muscles. J Int Soc Sports Nutr 9:26

Sale C, Artioli GG, Gualano B, Saunders B, Hobson RM, Harris RC (2013) Carnosine: from exercise performance to health. Amino Acids 44:1477

Swietach P, Youm JB, Saegusa N, Leem CH, Spitzer KW, VaughanJones RD (2013) Coupled $\mathrm{Ca}^{2+} / \mathrm{H}^{+}$transport by cytoplasmic buffers regulates local $\mathrm{Ca}^{2+}$ and $\mathrm{H}^{+}$ion signalling. Proc Natl Acad Sci 110:2064
Tillin NA, Pain MT, Folland JP (2013) Identification of contraction onset during explosive contractions Response to Thompson et al "consistency of rapid muscle force characteristics: influence of muscle contraction onset detection methodology" [J Electromyogr Kinesiol 2012; 22, 893]. J Electromyogr Kinesiol 23:991

Tran K, Smith NP, Loiselle DS, Crampin EJ (2009) A thermodynamic model of the cardiac sarcoplasmic/endoplasmic $\mathrm{Ca}^{2+}$ (SERCA) pump. Biophys J 96:2029

Westerblad H (2016) Acidosis is not a significant cause of skeletal muscle fatigue. Med Sci Sports Exerc 48(11):2339-2342

Westerblad H, Lännergren J, Allen DG (1997) Slowed relaxation in fatigued skeletal muscle fibers of xenopus and mouse. J Gen Physiol 109(3):385

Zaloga GP, Roberts PR, Nelson TE (1996) Carnosine: a novel peptide regulator of intracellular calcium and contractility in cardiac muscle. New Horiz 4:26 